\title{
Towards harmonizing approaches for scaling up access to arsenic safe water in Bangladesh: The Arsenic Safe Village Concept
}

\author{
N. Akter ${ }^{1}$, B. Onabolu ${ }^{1}$, M. Bolton ${ }^{2}$ \& H. Sargsyan ${ }^{1}$ \\ ${ }^{1}$ UNICEF Bangladesh, Dhaka, Bangladesh \\ ${ }^{2}$ Golder Associates Ltd., Canada
}

\begin{abstract}
Although 97.8\% of the population in Bangladesh haves access to improved water sources, 19.7 million people use water sources that are contaminated above the Government of Bangladesh (GOB) standards for arsenic (As, $50 \mu \mathrm{g} \mathrm{L}^{-1}$ ), posing a significant health risk. There were many initiatives taken by Government and donors on As mitigation, however the situation did not improve significantly. UNICEF Bangladesh with partners implemented a new approach of As mitigation by addressing entire villages to provide As-safe water and with improved sanitation and hygiene behavior. Rather than thinking about individual water points, consider the entire population in a village. The core objectives of this approach are a quantitative, measurable, reduction in As exposure, and the aim is to building up areas of contiguous As safety in the sequence of village to union, union to upazila and then to district. Using this approach 126 villages have been declared 'Arsenic-safe village with improved sanitation and hygiene'.
\end{abstract}

\section{INTRODUCTION}

According to the MICS 2012-2013 report, nationwide, 19.7 million people use water sources that are contaminated above the Government of Bangladesh (GOB) standards for arsenic (As, $50 \mu \mathrm{g} \mathrm{L}^{-1}$ ) and twice that number drink water with levels above the WHO guidelines $\left(10 \mu \mathrm{g} \mathrm{L}^{-1}\right)$.

Arsenic mitigation in Bangladesh has proceeded piecemeal, through installation of devices that serve $100-200$ persons or less, in villages of typically 2,000 5,000 people, resulting in a patchy distribution of safe water supplies. Various alternative water supplies and As removal technologies have been endorsed in the National Policy for Arsenic Mitigation (GOB, 2004) for use in As affected areas. Deep tube-wells have generally been the preferred option, as there have been operational challenges with the As removal technologies and sustainability is uncertain. Based on the technical challenges and scale of the As contamination, As mitigation has been neglected in some areas. In the absence of specific targets, for the country or individual agencies, progress since the mid-2000s stagnated. In the meantime, several million new wells have been installed; however, many have not been tested for As.

Despite the money and effort that has been committed, attempts to resolve the As issue have lost momentum and worse, As poisoning is becoming accepted as the inevitable fate of the Bangladeshi people. A comprehensive approach to As mitigation is required to address this crisis and to provide equitable access to all members in a community, including the most vulnerable.

\section{Village $\Rightarrow$ Union $\Rightarrow$ Upazila $\Rightarrow$ District}

Figure 1. UNICEF DPHE Protocol for scaling arsenic safety in Bangladesh.

\section{CONCEPT}

The UNICEF DPHE Protocol was used to implement an innovative approach to As-mitigation by intervening in entire villages and promoting sanitation and hygiene. Rather than thinking about individual water points, consider the entire population in a village and construct appropriate technologies to provide effective coverage based on the site-specific information and options. The aim is to build up areas of contiguous As safety in the sequence (Fig. 1).

\section{APPLICATION/ACTION}

From August 2012 to December 2014, with technical, financial and monitoring support from UNICEF, a project with the specific objective of "creating arsenic-safe model villages with sustainable improvement in water, sanitation and hygiene" was implemented in Comilla, Brahmanbaria and Narail districts. With technical assistance from Department of Public Health Engineering (DPHE) and Local Government Institutions (LGIs), UNICEF implemented this project in partnership of two national NGOs, Village Education Resource Centre (VERC) and Environment and Population Research Centre (EPRC). 


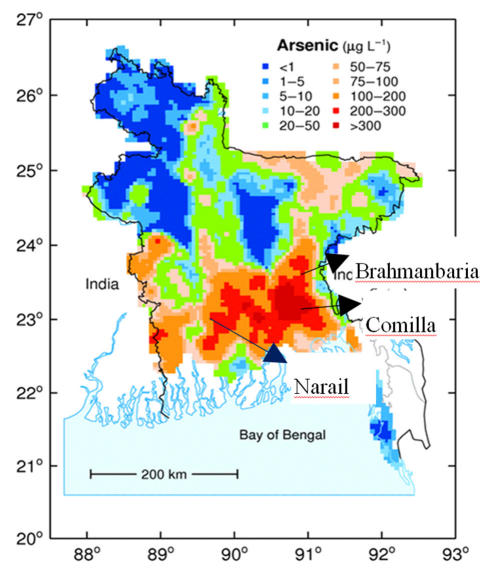

Figure 2. Targeting the high priorityintervention areas in Comilla based on vulnerability risk assessment.

\section{MAJOR COMPONENTS}

\subsection{Vulnerability risk assessment}

The project areas were selected on the basis of vulnerability risk assessment using ratio of As contamination, existing safe water coverage, number of arsenicosis patients and extreme poor. Selected 26 very high priority/high priority unions (DPHE and JICA, 2010) of Manoharganj upazila under Comilla, Banchharampur and Sarail upazilas under Brahmanbaria and Lohagora and Kalia upazilas under Narail District (Fig. 2).

\subsection{Community planning and leadership}

The Community Action Plan (CAP) is a key element of the project. The CAP process aims to assess the WASH situation in a community and to identify an appropriate approach to make improvements across the community, with special consideration to the needs of the poorest.

\subsection{Arsenic screening and monitoring}

To facilitate planning and target the most severely As affected people, all the tube-wells in the project areas were screened and a periodic monitoring system was established.

\subsection{Selection of appropriate technologies}

Based on the hydrogeological conditions and water quality considerations in the project areas, through community consultation different technological options are provided, including deep tube-wells, multiple connections to As-safe wells, rainwater harvesting systems, and Piped Water Supply Systems (Fig. 3).

\subsection{Water quality testing}

For each new water point, water quality testing is conducted with field test kits. $10 \%$ of the samples cross

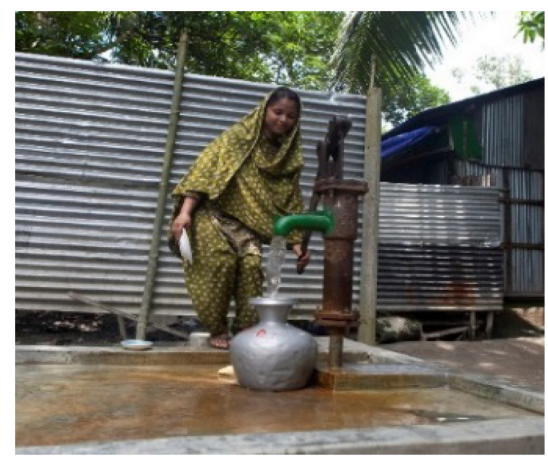

Figure 3. Collecting water from arsenic safe DTW.

checked in a laboratory. Following confirmation of acceptable water quality, water point is handed over to the community.

\subsection{Caretaker training and water safety plans}

Caretakers are trained and provided with tools for system operation and maintenance (O\&M) and water safety.

\subsection{Social mobilization for sanitation and hygiene education and School programmes}

Communities are informed about the ill-effects of drinking As-contaminated water, open defecation and unhygienic behavior through different social mobilization tools and techniques and sanitation promoted.

Arsenic safe water facilities installed and sanitation blocks are constructed (with running water, separate latrines for boys and girls).

\subsection{Arsenic-safe village declaration}

When all the households of a village receive access to As-safe water and ensure use of hygienic latrines, the village is declared as an 'As-safe village with improved sanitation and hygiene'.

\section{MAJOR OUTCOMES}

126 villages in three districts have been declared 'As-safe with improved sanitation and hygiene' and become models for replication in other affected areas of Bangladesh. The Department of Public Health Engineering and UNICEF Bangladesh are scaling up this concept in 15 unions (Sub-districts in Bangladesh).

\section{CONCLUSION}

The use of a community led demand responsive approach to provide As-safe water is a critical step 
towards harmonizing approaches for scaling up As mitigation in drinking water in Bangladesh.

\section{ACKNOWLEDGEMENTS}

We acknowledge German National Committee (UNICEF), the Embassy of the Kingdom of The Netherlands and the Swedish International Development Cooperation Agency (Sida).

\section{REFERENCES}

DPHE \& JICA 2010. Situation analysis of arsenic mitigation 2009. Local Government Division, Government of Bangladesh.

MICS 2012-2013. Bangladesh Multiple Indicator Cluster Survey 2012-2013, Progotir Pathey: Final Report. Bangladesh Bureau of Statistics (BBS) and UNICEF Bangladesh, 2014, Dhaka, Bangladesh. 